\title{
THE ASSOCIATION BETWEEN NIGHT SHIFT WORK AND NUTRITION PATTERNS AMONG NURSES: A LITERATURE REVIEW
}

\author{
Beata Pepłońska ${ }^{1}$, Paulina Nowak², Elżbieta Trafalska ${ }^{3}$ \\ ${ }^{1}$ Nofer Institute of Occupational Medicine, Łódź, Poland \\ Department of Environmental Epidemiology \\ ${ }^{2}$ Polish Social Insurance Institution, Second Branch in Łódź, Łódź, Poland \\ ${ }^{3}$ Medical University of Lodz, Łódź, Poland \\ Department of Nutrition Hygiene and Dietetics
}

\begin{abstract}
The shift work system may affect the temporal distribution of eating and diet quality. The paper aimed at reviewing a body of research examining the associations between night shift work and dietary habits among nurses. Data from the PubMed and Google Schoolar databases, as well as references lists in selected papers were searched. The authors used the following keywords: nurses, shift work, diet, nutrition. Papers published in English or Polish were selected for the review, and as many as 19 papers published in 2000-2017 were eventually identified. The studies varied greatly with respect to the study size, subjects' age and the duration of night shift work. The major problem was the heterogeneity of the tools used for dietary assessment. Self-administered questionnaires were used and analyses were rarely adjusted for confounders. Alcohol consumption was the most frequently analyzed aspect ( $\mathrm{N}=8$ studies), followed by the total energy $(\mathrm{N}=7)$, protein, fat $(\mathrm{N}=6)$, and carbohydrate intake, coffee and fruit consumption $(\mathrm{N}=5)$. The results showed quite a consistent association of night work with higher coffee (caffeine) consumption, as well as lower alcohol, and fruit and vegetables consumption. Few studies also reported more frequent snacks consumption, later time of the last meal, eating at night, meals irregularity, and a poorer diet quality among night shift nurses when compared to the reference. The review showed some poor nutritional habits among nurses working night shifts. However, the topic warrants further attention, owing to the relatively small number of the studies performed so far, and their numerous methodological limitations. Med Pr. 2019;70(3):363-76
\end{abstract}

Key words: shift work, lifestyle, nurses, circadian rhythms, nutrition, night work

Corresponding author: Beata Pepłońska, Nofer Institute of Occupational Medicine, Department of Environmental Epidemiology, św. Teresy 8, 91-348 Łódź, Poland, e-mail: beata.peplonska@imp.lodz.pl

Received: September 13, 2018, accepted: November 22, 2018

\section{BACKGROUND}

About $20 \%$ of the workforce in general work night shifts [1]. The shift work system, and night work in particular, is frequently found in health care, due to the 24-h medical service required for hospital patients. Nurses' work frequently involves shift work and night work.

Many epidemiological studies conducted among shift workers have shown a higher risk of chronic diseases, including cardiovascular disease, diabetes, metabolic syndrome or even cancer [2]. Moreover, such conditions as sleep disorders [3] and obesity [4] are more frequently observed among night shift workers than among day workers. Circadian rhythm disruption has been proposed as one of the possible underlying mechanisms [3].
Among the issues closely related to irregular working hours is the irregularity of food consumption. However, this abnormal circadian timing of meals may have adverse effects on the processes of digestion, nutrient absorption, enzyme activity, and metabolism, as well as the sensations of hunger, appetite and satiety [5].

The association between the shift work system and nutrition has been investigated since the mid-1960's. Lowden et al. identified as many as 21 research articles published between 1967-2009 that reported on studies concerning a possible relationship between shift work and dietary habits [6]. They concluded that shift work significantly affected the temporal distribution of eating, diet quality, and energy distribution over the course of the day. The authors of another review covering the

Funding: This research was supported by the Nofer Institute of Occupational Medicine (statutory institutional grant No. 10.32/2017 entitled: "The association between night shift work and nutrition patterns of nurses and midwives," principal investigator: Beata Pepłońska, M.D., Ph.D.). 
period of 1990-2007 noted that in most of the 7 studies identified (including 2 conducted among nurses) some adverse effects on nutrition were observed, such as more frequent and poor quality meals [7]. The most recent review of research in this field [8] has also pointed out a poorer diet quality among shift workers. The reviews have identified certain methodological limitations of the studies, such as small population samples, the use of less-valid dietary assessment methods, or the lack of comparisons with the non-exposed, reference group of day workers $[6,8]$.

To the best of the authors' knowledge, no review of the studies devoted to the assessment of shift work and night work among nurses, and their nutrition habits, have been performed to date. The shift work of nurses is usually organized according to the fast rotating scheme, and it often involves night work. It can be assumed that the nurses' professional background implies a better understanding of health-related issues, including also those related to healthy diet and nutrition, as compared to that expected in the general population and among blue collar workers performing shift work. Some specific factors, like an insufficient time for rest or stress, may also influence dietary choices in this professional group. Therefore, it can be presumed that the conclusions from the previously reported reviews may not directly apply to nurses.

In the present report, the authors aimed at reviewing the research devoted to the associations between night shift work of nurses and their dietary habits, seeking to determine whether any improper behaviors might be the case.

\section{MATERIAL AND METHODS}

The literature review was based on data from the PubMed and Google Scholar databases, as well as on the citations search performed in selected papers. The following combinations of keywords: "nurses and shift work and diet" or "nurses and shift work and nutrition" were used. Papers published in English or Polish were selected for the review, and only those that concerned the differences in nutrition patterns by the system of work among nurses were included. Eventually, as many as 19 publications were identified in this field.

\section{RESULTS}

The basic characteristics of the studies that were subject to review, including study design, population size, shift work characteristics, diet characteristics and major findings, are displayed in Table 1.

The studies are presented in chronological order, with the focus on methodological aspects.

The study by Geliebter et al. included 85 subjects: nurses, nurses aids and security personnel [9]. The differences in dietary characteristics were compared between late shift (evening and night) workers and day workers. The study focused on the times and number of main meals, time of the last meal, and whether daily food intake changed since the start of the current shift. The study showed that the late shift workers reported fewer meals than the day workers, and a later hour of the their last daily meal. A trend could also be noted for the late shift workers to eat more since the beginning of the later shift. The analysis included age, years of shift work and smoking as covariates. The study was limited by a small population size and the use of an unvalidated questionnaire. Moreover, the night shift and evening shift workers were combined into 1 category; consequently, the differences in effects between these 2 groups could not be distinguished.

Reeves et al., in their study assessing the dietary habits of 36 subjects who were the male and female staff of nursing homes and hospitals, used 6-day food diaries [10]. The researchers also asked questions about the number of main meals and snacks. The data collected were then analyzed using Diet 5 for Windows, which allows for determining the total energy, carbohydrate, fat and protein intake, as well as alcohol consumption. No differences between the night shift and day workers were observed in terms of the total energy consumption. The night shift workers reported consuming food during night hours while on night duty. Women working night shifts were more likely to consume fewer meals but more snacks per day than day workers. Moreover, they reported drinking more coffee and tea cups per day than female day workers. The study was limited by its small population size and crude comparisons.

The study by Bilski et al. was conducted among 241 female nurses (171 working night shifts), and their dietary habits were evaluated based on a set of questions inquiring about health behaviors during the night shift [11]. The analysis showed that only $9.9 \%$ of nurses had a hot meal during the night duty, which was usually some stodgy food, and as many as $9.9 \%$ reported drinking only coffee during the night duty. The overall daily coffee consumption was higher among the night shift workers although no statistics were presented. The study was limited by the lack of comparisons between 


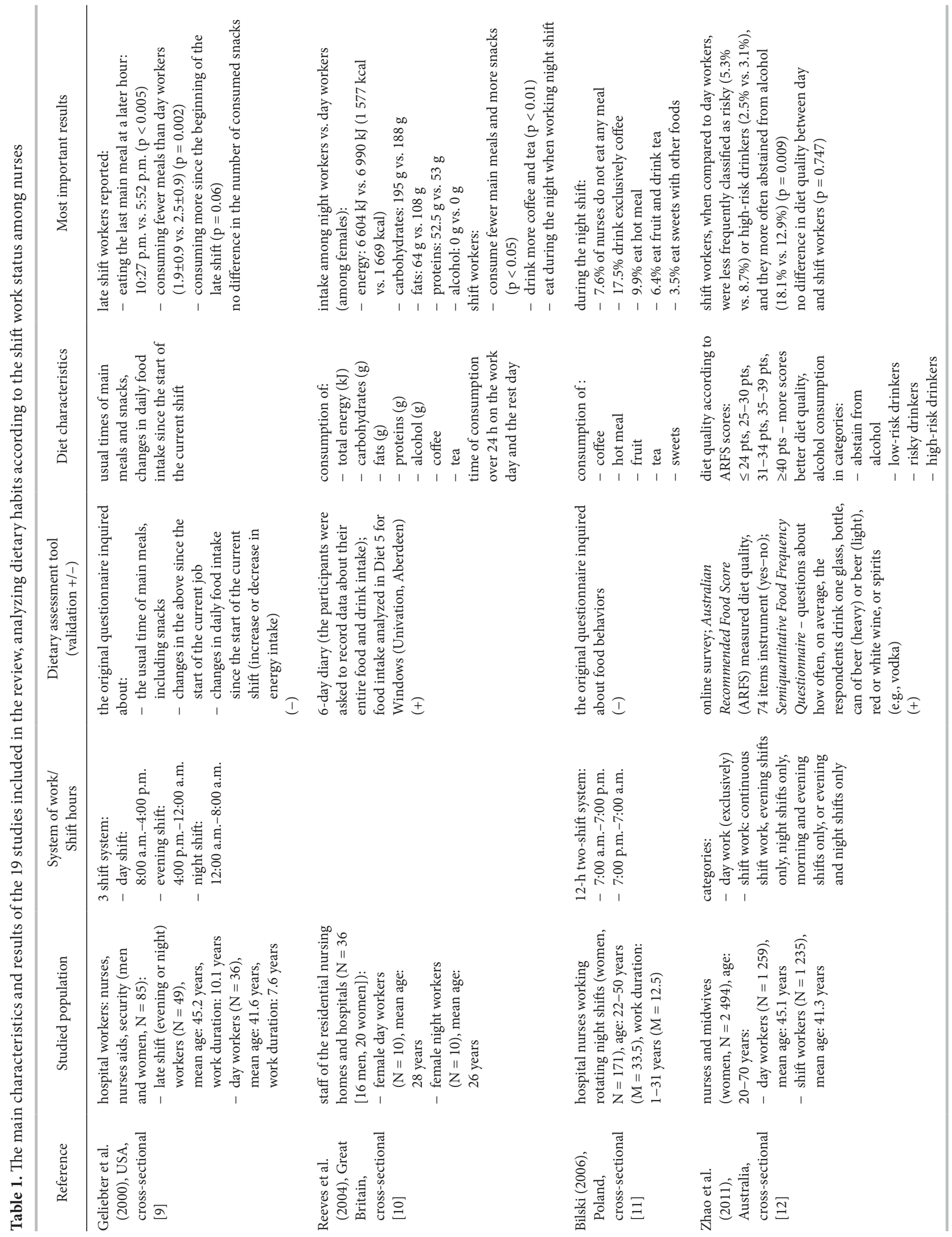




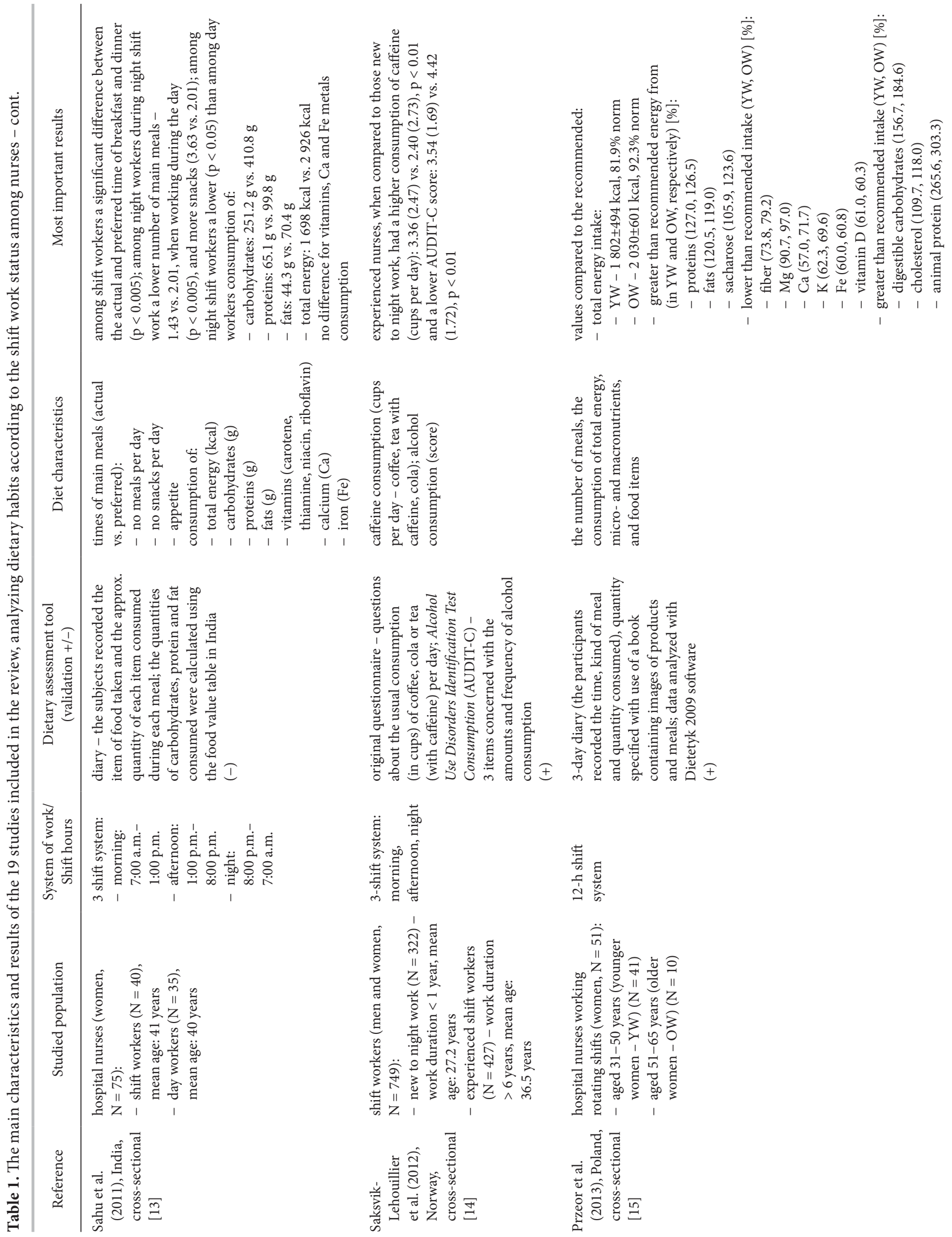



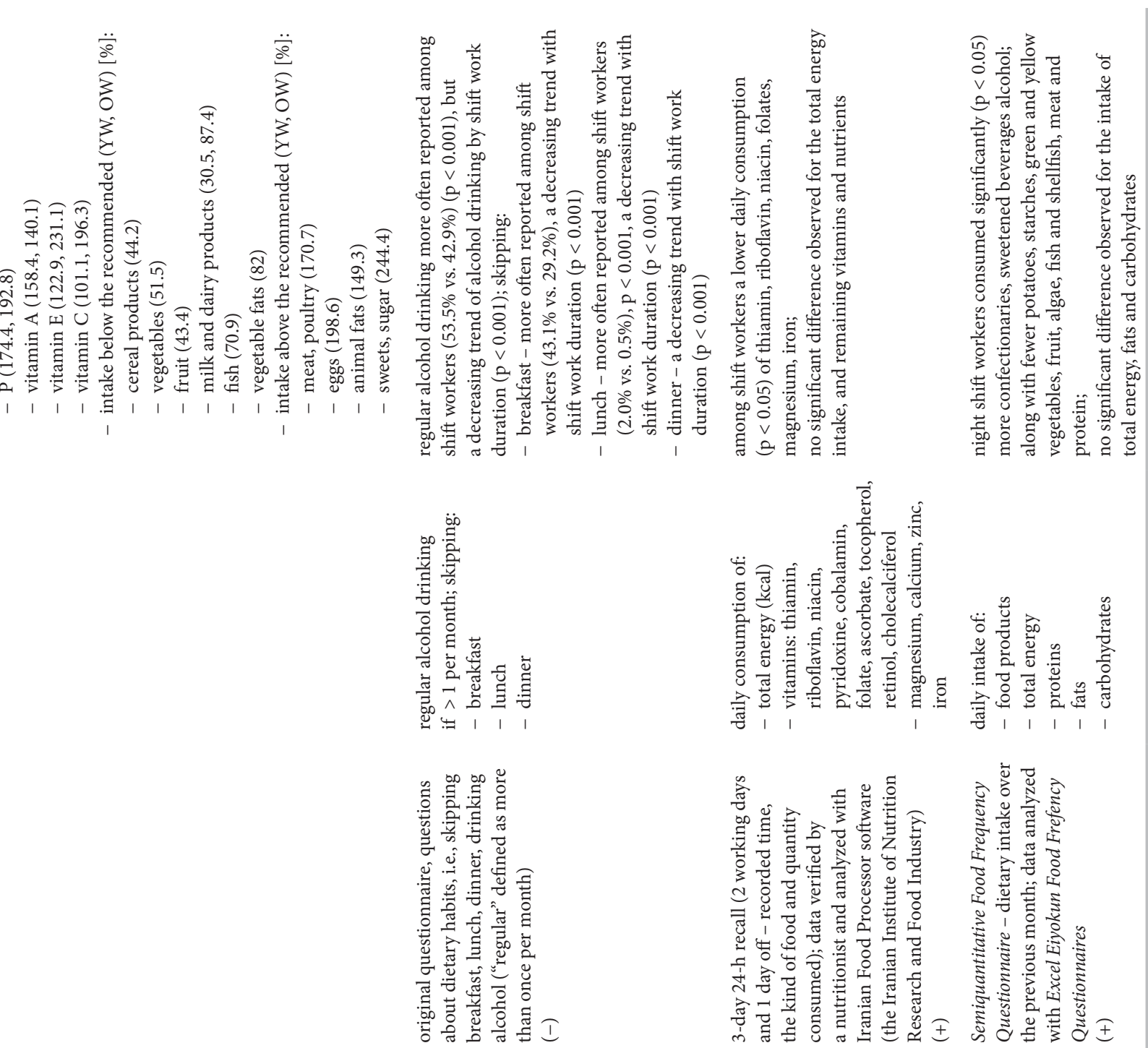

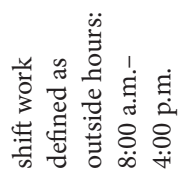
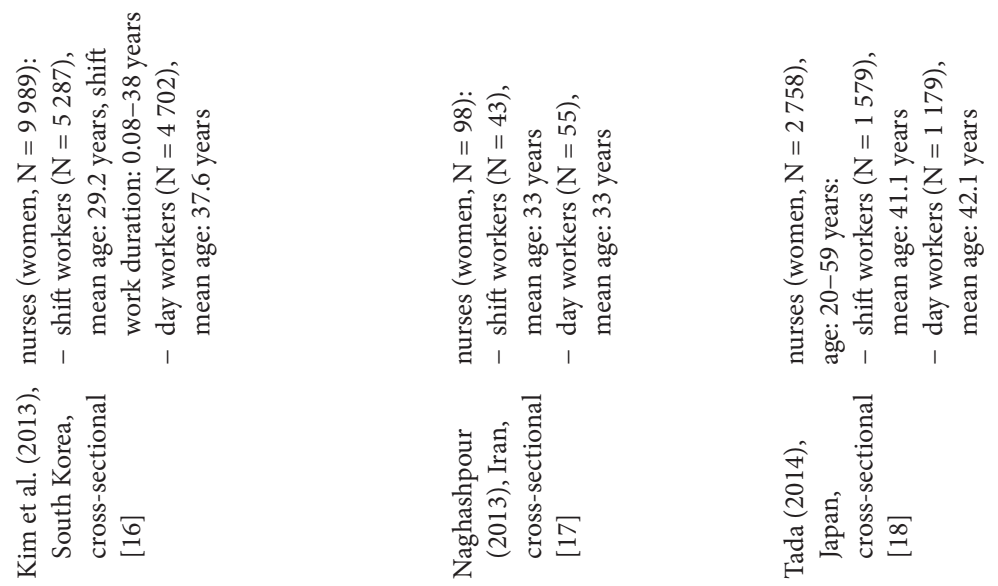


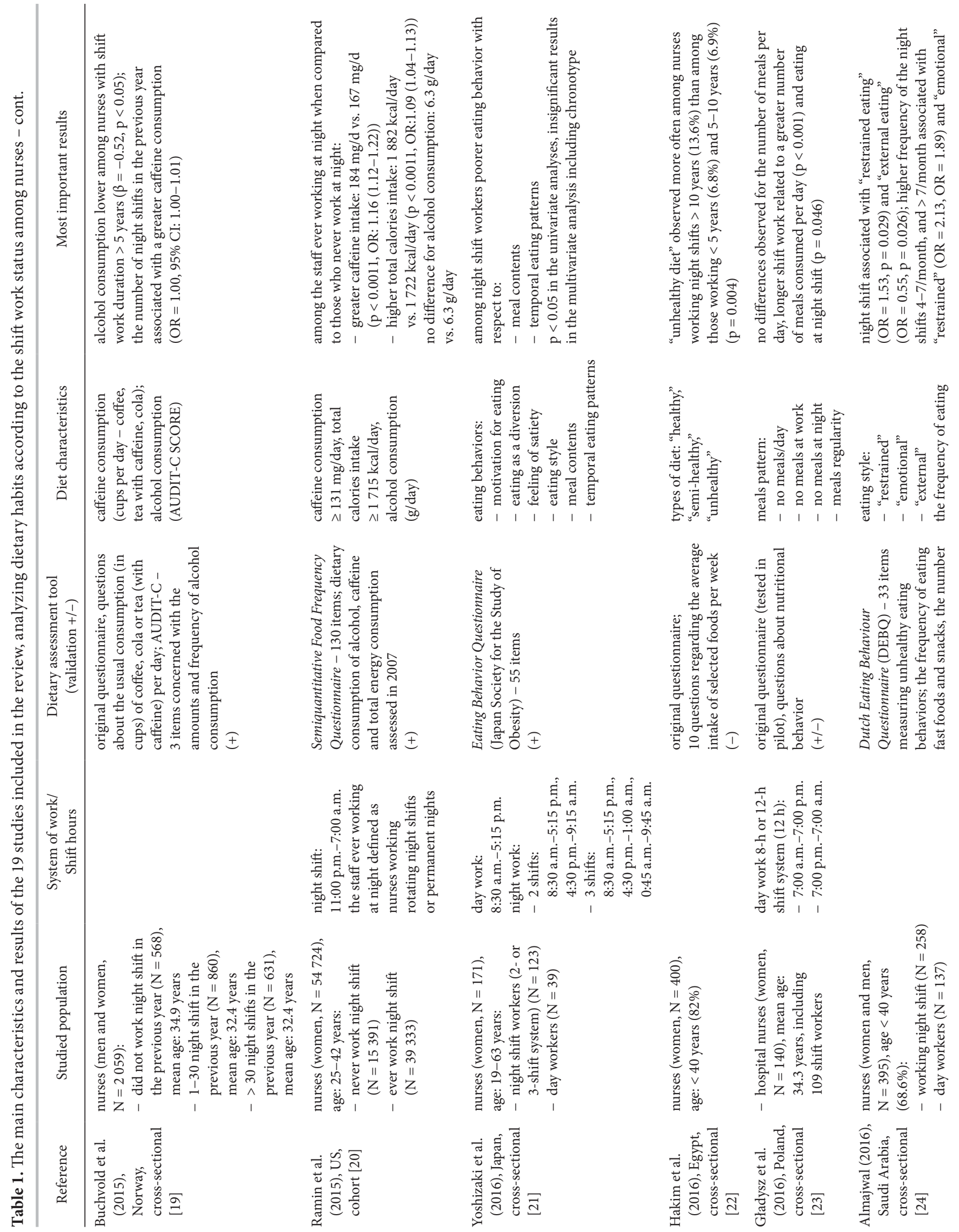



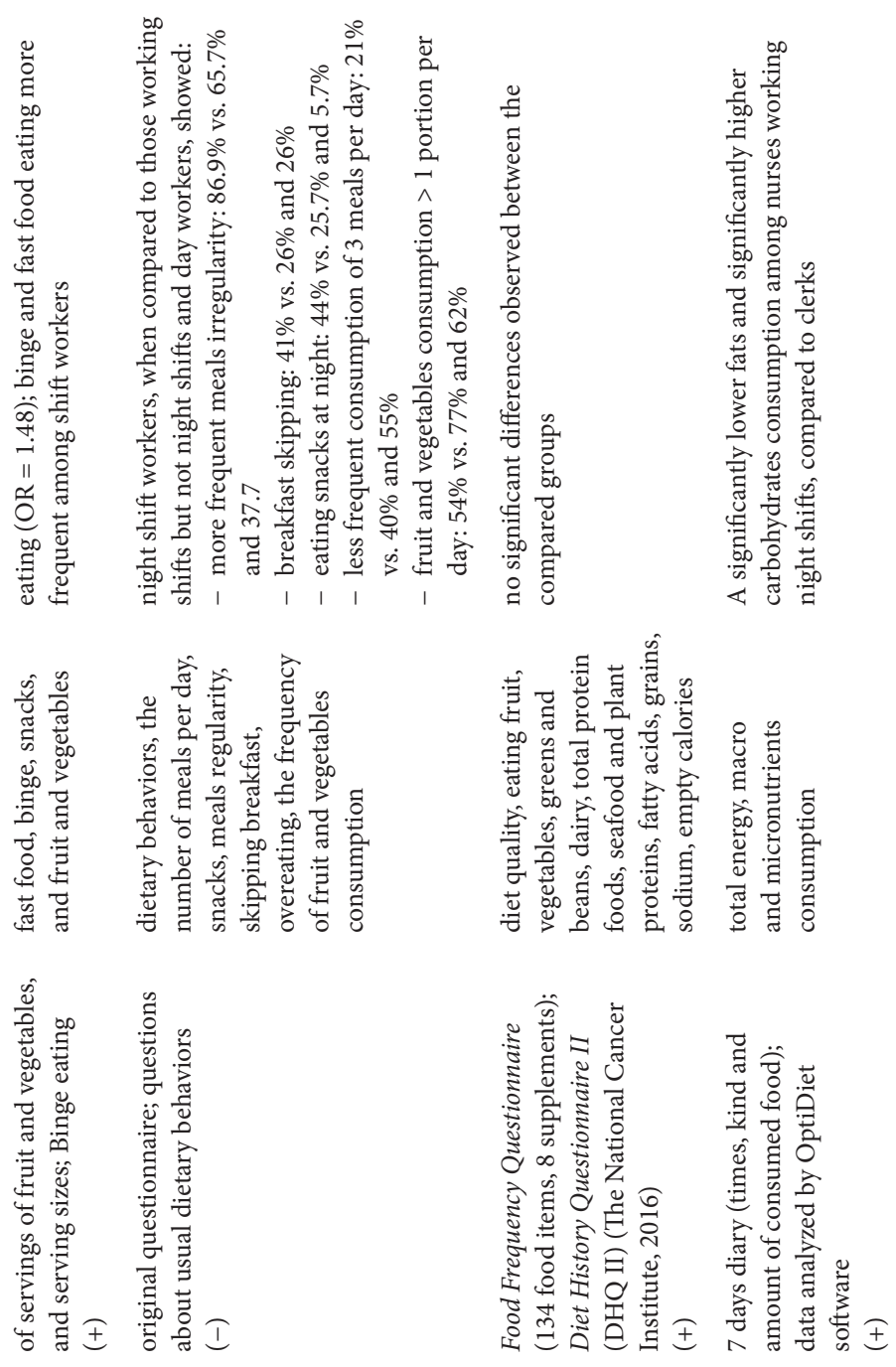

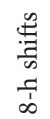
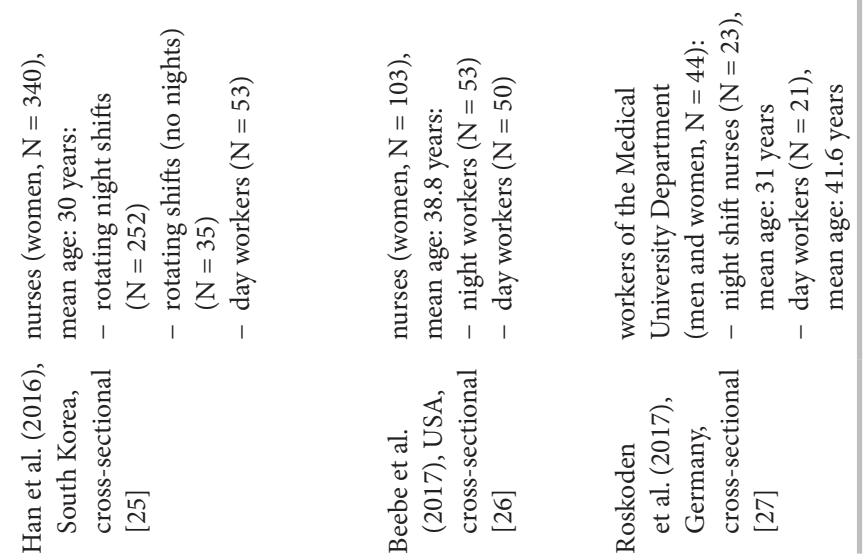

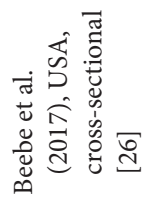

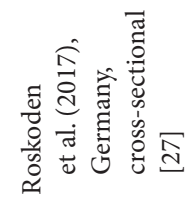


night shift and day workers. Moreover, the questionnaire used was developed by the researchers themselves and no information on its validation was provided.

Zhao et al. analyzed diet quality and alcohol consumption in an e-cohort of 2494 nurses and midwives [12]. Diet quality was measured using Australian Recommended Food Score (ARFS), which is a 74-item validated instrument. The average alcohol consumption was assessed with semiquantitative FFQ, and the subjects were classified into 4 categories: abstaining from alcohol, lowrisk drinkers, risky drinkers and high-risk drinkers. The analysis showed no differences in diet quality between the day and shift workers. The latter more frequently reported abstaining from alcohol, and were less frequently classified as risky or high-risk drinkers. The difference in the distribution was statistically significant, and the analysis was adjusted for age.

A group of 40 female hospital nurses and 35 day working nurses were studied in India [13]. Their dietary habits were assessed based on data from diaries in which the subjects recorded the specific food items and amounts consumed. Food value tables were used to calculate total energy, protein and carbohydrate intake, as well as some vitamin and mineral intake. The average values of the last 2 items were calculated on the basis of 15 days consumption data. According to the study results, the night shift workers reported to consume fewer main meals but more snacks during the day with the night duty, when compared to the day workers. They were also found to have a lower energy, protein, fat and carbohydrate intake. No difference was observed for vitamin or mineral consumption. The subjects in both groups were of similar age, and the night shift workers were slightly slimmer than the day workers. Only crude analyses were performed in this study.

A population of 749 Norwegian nurses working night shifts was investigated by Saksvik-Lehouillier et al. [14]. The study population included 322 women with less than 1 year of experience in night shift work and 427 with over 6 years of respective experience. During the study, the researchers inquired about the number of coffee, coke or tea cups usually consumed daily. Alcohol consumption was measured with Alcohol Use Disorders Identification Test Consumption (AUDIT-C), a validated tool. The crude comparison performed showed a significantly higher coffee consumption and a lower AUDIT-C score among more experienced nurses.

A cross-sectional study on a population of 51 hospital nurses working night shifts was carried out in Poland [15]. The nurses completed a diary reporting their food consumption over a 3-day period: before the night duty, during the duty and on the day off. The dietary data (total energy, daily macro- and micronutrient consumption) were analyzed with computer software. The study results revealed lower than recommended values of the total energy consumption, and those of fiber, $\mathrm{Ca}$, $\mathrm{Mg}, \mathrm{Fe}, \mathrm{K}$, vitamin $\mathrm{D}$, folic acid, and fluid intake, and a higher intake of animal proteins, fat, cholesterol, $\mathrm{Na}, \mathrm{P}$, $\mathrm{Zn}, \mathrm{Cu}$, and vitamins $\mathrm{A}, \mathrm{E}, \mathrm{B}$ and $\mathrm{C}$. This finding was associated with a lower consumption of cereals, fruit, vegetable, dairy products, processed meat products, fish, and vegetable fats, and a higher than recommended consumption of meat, poultry, eggs, animal fats, sweets, and sugar. In this study, no formal reference group was established. Instead, the calculated values were compared with recommended standards, and the results indicated potential disparities. The findings pointed to a lower than recommended energy intake, but at the same time, the BMI for some subjects was at the upper limit value or even indicated overweight, which would imply a dietary underreporting.

A large cross-sectional study of 9989 Korean nurses was carried out by Kim et al., who used the web-based survey [16]. A self-administered questionnaire included questions on alcohol consumption and dietary habits. Night shift nurses reported a higher consumption of alcohol when compared to day workers, but the frequency of a regular drinking habit decreased with an increasing duration of shift work. The shift work nurses more frequently reported skipping breakfast and/or lunch, but this tendency was found to significantly decrease with an increasing duration of shift work. The crude analyses performed did not include any adjustments for covariates.

The cross-sectional study to assess dietary habits among 98 Iranian hospital nurses employed 24-h recalls of a 3-day period [17]. The dietary data were verified by a nutritionist. The total energy, vitamin and micronutrient intakes were calculated. The data analysis showed that shift workers consumed less thiamin, riboflavin, niacin, folates, magnesium, and iron. No differences between the shift and day workers were observed with respect to daily consumption of total energy, pyridoxine, cobalamin, ascorbate, tocopherol, retinol, cholecalciferol, zinc, and calcium. The crude means were compared using Student's t-test.

Dietary intake over a 1-month period was evaluated in a cross-sectional study of 2758 nurses in Japan [18]. In this study, a semiquantitative food frequency questionnaire was used and total energy consumption, as well as 
product groups and nutritional elements, were evaluated. Women working shifts reported a higher consumption of sweets, sugar-sweetened beverages and alcohol, and a lower consumption of potatoes and starch, green and yellow vegetables, white vegetables, fruit, algae, fish and shellfish, and meat. Protein consumption was significantly lower among the shift workers, but no difference was observed for the intake of total energy, fats and carbohydrates. The analysis was adjusted for total calories intake.

A cross-sectional study of 2259 Norwegian nurses investigated the association between night shift work duration ( $>5$ years vs. $<5$ years) and its intensity, expressed as the number of night shifts over the previous year, and daily caffeine consumption and alcohol consumption [19]. Alcohol consumption was measured using AUDIT-C. The study found a statistically significant inverse association between a longer duration of night shift and alcohol consumption. A higher number of night duties during the year preceding the study was significantly associated with coffee consumption. The multivariate analysis included age, gender, the average number of working hours and cohabitating children as covariates.

In a large Nurses Health II cohort study of 54725 women (39 333 ever working night shifts), the authors examined the association between shift work and total energy intake, as well as coffee and alcohol consumption [20]. Dietary data were collected via a validated semiquantitative food frequency questionnaire covering 130 food items. For the logistic regression analysis, the total energy consumption was classified into 2 categories: $\geq 1712 \mathrm{kcal} /$ day and $<1712 \mathrm{kcal} /$ day, and coffee consumption $\geq 131 \mathrm{mg} /$ day and $<131 \mathrm{kcal} /$ day. The analysis showed a statistically significant association between higher calories and coffee consumption, and ever working night shifts. The multivariate analysis included a number of covariates, such as age, partner's education, smoking, physical activity chronotype, and BMI. The basic characteristics of the study population did not show any differences in alcohol consumption due to the night shift status.

Yoshizaki et al. investigated dietary habits in a group of 162 nurses (including 123 working night shifts) [21]. The dietary behaviors were measured with the self-administered questionnaire. The questionnaire used was developed by the Japan Society for the Study of Obesity and employed a scoring system for the measurement of obesity-related behaviors such as cognition of constitution, motivation for eating, eating as a diversion, feeling of satiety, eating style, meal contents and temporal eating patterns. The crude data analysis showed that the night shift workers had a more unbalanced diet with a greater preference for high-fat diet and sweets, and an abnormal eating pattern, including a greater irregularity in the timing and number of meals, and later time of meals. The multivariate analysis that included age, marital status, residential status, smoking, alcohol status, and the number of night shifts during the previous month revealed an association with the chronotype but not with the night shift work.

Hakim et al. investigated nutritional behaviors among 400 nurses, considering the duration of night shift work [22]. They used a 10-item questionnaire to inquire about the usual frequency of particular food item consumption per week. Dietary data were analyzed based on the scoring system, categorizing women into 3 groups of 'healthy', 'semi-healthy' and 'unhealthy' diet. The crude analysis of group distribution according to shift work duration showed a more frequent 'unhealthy' diet in the group of nurses working night shifts for a period of more than 10 years. These differences were not statistically significant.

The nurses' dietary habits at the workplace were examined in a study of 140 hospital nurses in Poland (including 109 shift work nurses) [23]. In this study, a questionnaire developed by the study authors was used to elicit data on the number of meals per day, the number of meals while at work, main meals, meals regularity, and eating at night. Nurses with a history of night shift work of more than 20 years reported consuming more meals per day than the nurses who had a shorter history of night shift work. Nurses working night shifts and those who had a 12-h duty but only during the day, were more frequently found to have meals at night than the nurses who had a 8-h day time duty. The findings were analyzed using crude $\chi^{2}$ test unadjusted for any covariates.

A population of 395 nursing staff at 2 hospitals in Saudi Arabia was investigated in a cross-sectional study by Ajmawal [24]. The Dutch Eating Behaviour Questionnaire, a validated tool comprising 33 questions, was used to categorize subjects into the following categories: restrained (a tendency to restrict food intake to control body weight), emotional (to cope with negative emotions) and external eating (the extent to which external cues of food trigger the eating episodes). The author found a statistically significant positive association between night shift work, particularly a higher frequency of night shifts per month, and restrictive or emotional 
eating, and an inverse association with external eating. The analyses included age, gender, education, and marital status as covariates.

Han et al. conducted a cross-sectional study of 340 nurses in South Korea, evaluating their dietary behaviors, the number of main meals, snacks, meals irregularity, skipping breakfast, overeating, and the frequency of fruit and vegetable consumption [25]. The questionnaire inquired about the items that matched the recommendations of the Korean Dietary Guidelines. The night shift workers more frequently than the day workers reported meals irregularity, skipping breakfast, and eating snacks at night, while less frequently eating 3 main meals per day and more fruit servings per day. The crude comparisons did not include any covariates.

Diet quality and night shift work were evaluated among 103 hospital nurses in the USA (53 working night shifts) [26]. Diet History Questionnaire II (DHQII) developed by the National Cancer Institute was used to ask questions about the frequency and amount of consumption of 134 food items and 8 dietary supplements over a period of one month preceding the study. The findings revealed no differences in diet quality of the night shift nurses when compared to the day nurses. The crude analysis did not account for any covariates.

Nutritional habits were evaluated in a small group ( $\mathrm{N}=46)$ of medical university staff (23 nursing staff working night shifts, 10 nurses working during the days, and 13 clerical staff) in Germany [27]. The information about the foods consumed was collected via diaries covering a 7-day period. Data were analyzed with OptiDiet software to calculate macro- and micronutrient intake. In this study, no differences in the total energy intake were found between night shift and day workers. Both the nurses working night shifts and those working only during days consumed significantly less fats than did the clerical staff. Carbohydrate intake among the night shift nurses was significantly higher than among the office staff, but not when compared to the day nurses. No covariates were included in the crude analyses of the data.

\section{CONCLUSIONS}

The aim of the present review was to evaluate the existing epidemiological data on the association between shift work, with special regard to night shift work, and the nutritional habits of nurses working according to that pattern. The authors have identified altogether as many as 19 papers describing studies in this area, which were published in 2000-2017. The total number of studies in this field is relatively small, but an increasing interest has been noted over the last decade, and as many as 16 of the 19 papers identified have been published since 2011.

The studies varied greatly with respect to the size and age of the study populations, and the duration of night shift work. The major problem which could be identified is the heterogeneity of the tools used for dietary assessment. Therefore, the data aggregation, as well as direct comparisons and meta-analyses, are precluded at this stage.

Table 2 presents the findings of the studies reviewed, by the dietary items or behaviors examined, providing the number of studies that evaluated a specific item, and referencing the studies that found the consumption higher, lower or equal among shift work nurses when compared to the reference. Alcohol consumption was the most frequently analyzed (8 studies), followed by the total energy (7 studies), protein, fat (6 studies), and carbohydrate intake, as well as coffee and fruit consumption which were evaluated in 5 studies, i.e., in about one-third of the studies covered by this review. The other food items were examined even less frequently.

The results were fully consistent with respect to coffee (caffeine) consumption, indicating a more frequent or higher coffee consumption among the nursing staff working night shifts $[10,11,14,19,20]$. Coffee consumption seems to be related to a longer duration and a higher frequency of night shift work $[14,19]$. Two studies reported the consumption of more than 3 cups of caffeine drinks per day as being related to both a higher number of the night duties worked in the previous year and to night shift work seniority $[19,28]$. Dietary recommendations generally accept coffee consumption of up to 3 cups per day. Coffee helps overcome sleepiness and fatigue, and this may explain its higher consumption among night workers. The recent umbrella review of the observational and interventional studies of coffee consumption and any health outcomes has concluded that coffee drinking seems safe within the usual patterns of consumption except for pregnant women and women with an increased risk of fractures [29]. It should be borne in mind that the drinks containing coffee or caffeine may disturb the sleep pattern [30], with frequent awakenings and prolonged sleep latency. Thus, the sleep effectiveness and night work tolerance may decrease in the long run.

Given the increasing evidence on a higher risk of obesity among night shift workers, the data on the total energy intake are of particular interest. Among 
Table 2. Food items from observational studies analyzing dietary habits according to the shift work status of nurses

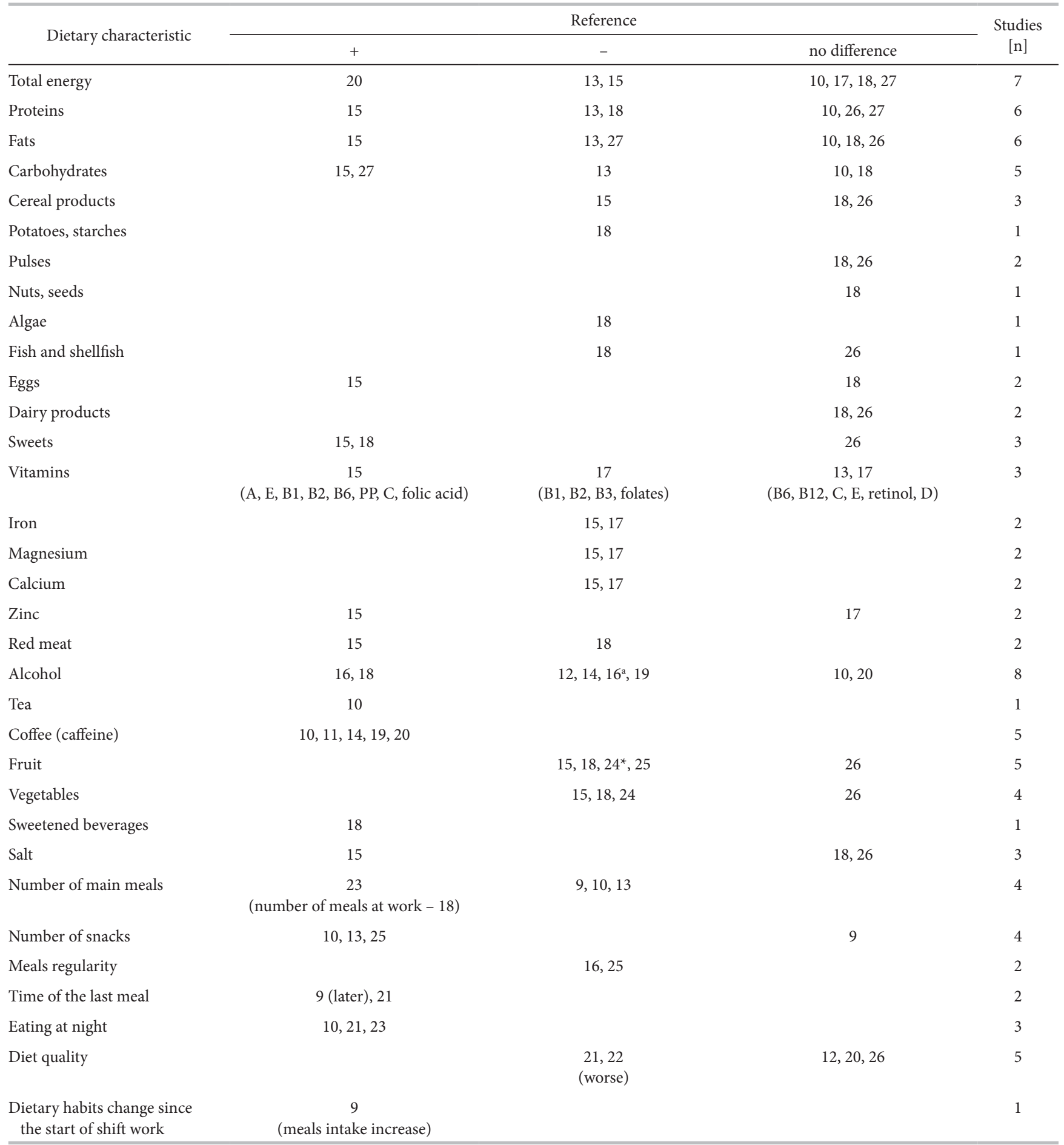

a Among shift workers with longer shift work duration.

* In the subgroup of emotional eating.

" + " - greater consumption compared to the reference group.

"-" - lower consumption compared to the reference group.

the 7 studies that evaluated the total energy intake by the shift work status, 4 studies reported no difference $[10,17,18,26,27], 1$ reported a higher energy consump- tion among shift workers [20] and 2 indicated a lower consumption $[13,15]$. Most of these studies were smallscale, and thus they may have been underpowered to 
elucidate the true inferences $[10,13,15,17,27]$. Of the 2 large and good-quality studies, 1 reported a positive association between "ever" working night shift and a higher total energy consumption [20], while the other revealed no difference for the current work schedule [18]. The differences in the definitions of exposure adopted in these studies, as well as those in ethnicity of the study population, could potentially explain the observed inconsistency in the study findings.

In addition, 2 out of 3 studies indicated a higher consumption of sweets or sweetened drinks [15,18], which could be related to the higher carbohydrate intake reported in 2 studies $[15,27]$. It has to be noticed, however, that in 2 out of 5 studies in total, where this parameter was calculated, no differences attributed to the system of work $[10,18]$, or even a lower carbohydrates intake [13] among the night shift workers, were found. The findings of a single small-scale study showed that shift workers reported eating more since the beginning of the later shift [9], but this observation was not replicated in other studies. For the body weight regulation, some other aspects, apart from the total energy intake, may also play a role. These include diet quality and composition, and temporal distribution of foods. Five of the studies analyzed diet quality $[12,20-22,26]$ using various questionnaire tools and guidelines. A poorer diet quality among night shift nurses was found in 2 studies [21,22], while 3 others revealed no differences in the analyses by the system of work $[12,20,26]$. Few studies indicated the specific aspects of poor dietary behaviors of nurses working night shifts, such as a lower number of main meals per day $[9,10,13]$, meals irregularity $[16,25]$, a later hour of the last meal $[9,21]$, and eating at night $[10,23]$. Similar problems have been indicated among shift workers in industry [6,7].

Although the number of studies that examined the intake of fruit and vegetables was relatively small, most of them reported lower consumption among night shift workers as compared to the reference $[15,18,24$, 25]. This adds to a poorer diet quality of the night shift workers.

There was a good consistency of the study results regarding alcohol consumption, which was found to be lower among night shift workers when compared to day workers $[12,14,16,19]$. Also, the duration of shift work tended to be associated with a lower alcohol consumption $[14,16,19]$.

The intake of vitamins and microelements such as iron, magnesium, zinc, and calcium, was rarely evaluated. Two of such studies reported a lower intake among nurses working night shifts as compared to the reference group $[15,17]$. These results derive from small-scale studies that employed the method of completing a diary on the foods consumed over a given period. Therefore, further studies are warranted to confirm these findings.

\section{Methodological issues}

The studies focused on the association between shift (night) work of nurses and their nutrition patterns had a cross-sectional design. While cross-sectional studies provide a good picture of the health care needs of the population at a particular point in time, their value in investigating etiological relationships is limited since the time sequence is unknown.

In the majority of the studies, the nursing staff have been investigated. In 2 studies [9,27], the populations were more heterogeneous, including nurses aids, security staff [9] and clerks [27]. As many as 13 out of 19 studies included only women, and 6 studies included subjects of both genders $[9,10,14,19,24,27]$. There were 6 small-scale studies, i.e., of less than 50 subjects per group $[9,10,13,15,17,27]$, while 8 studies included from several dozen up to 1000 subjects $[11,14,21-26]$. There were 4 studies examining relatively large populations of several thousand people $[12,16,18,19]$, and in 1 study, the population size was very large, of over 1000 [20].

The instruments used for dietary assessment are briefly characterized. In all the studies, the questionnaires were self-administered, which implies a possibility of some inaccuracies and underreporting [31]. Measurement error is inherent in any self-reported dietary assessment [32]. There was a large heterogeneity of the questionnaires used. The Food Frequency Questionnaire was used in 4 studies $[12,18,20,26]$. This tool aims at assessing the usual diet, and is much more precise than diaries. The diary method covering the periods from 3 days up to 15 days [13] was used in 5 studies $[10,13,15,17,27]$. Dietary diaries in which the subjects record the timing and food items consumed shortly after the meal were demonstrated to be reliable and valid tools [33]. However, they may not represent the usual diet, especially when covering a smaller number of days, and they do not consider seasonality.

Most frequently, the questionnaires developed by the authors of the research themselves were used [9, $11,14,16,19,22,23,25]$. In most cases, the tools were validated, but in the case of 6 studies, the validation was either not described or not performed $[9,11,13,16$, $22,25]$. 
It also has to be underlined that the analyses performed for the studies were usually not controlled for confounders. In fact, the adjusted analyses with control for various confounders were carried out only in 5 studies [18-21,24].

To conclude, the existing epidemiological evidence on the relationship between night shift work of nurses and their dietary habits is inadequate to draw any definite conclusions. The number of studies performed has been relatively small, and some methodological issues have not been properly addressed thus far. The picture is further complicated by the inconsistency of the results. However, the findings of the present review indicate that the major problem among nurses working night shifts may be a higher than usual coffee consumption, along with meals irregularity and improper timing that is inconsistent with the circadian clock. Further studies in this area are warranted, in particular on larger populations and using validated tools for the dietary assessment.

\section{REFERENCES}

1. Straif K, Baan R, Grosse Y, Secretan B, El GF, Bouvard V, et al. Carcinogenicity of shift-work, painting, and fire-fighting. Lancet Oncol. 2007;8(12):1065-6.

2. Wang XS, Armstrong ME, Cairns BJ, Key TJ, and Travis RC. Shift work and chronic disease: The epidemiological evidence. Occup Med (Lond). 2011;61(2):78-89.

3. Boivin DB and Boudreau P. Impacts of shift work on sleep and circadian rhythms. Pathol Biol. (Paris). 2014;62(5):292-301.

4. Peplonska B, Bukowska A, and Sobala W. Association of rotating night shift work with BMI and abdominal obesity among nurses and midwives. PLoS One. 2015;10(7):e0133 761, https://doi.org/10.1371/journal.pone.0133761

5. Haus E, Smolensky M. Biological clocks and shift work: Circadian dysregulation and potential long-term effects. Cancer Causes Control. 2006;17(4):489-500.

6. Lowden A, Moreno C, Holmback U, Lennernas M, Tucker P. Eating and shift work-effects on habits, metabolism and performance. Scand J Work Environ Health. 2010; 36(2):150-62.

7. Amani R, Gill T. Shiftworking, nutrition and obesity: Implications for workforce health - A systematic review. Asia Pac J Clin Nutr. 2013;22(4):698-707, https://doi.org/10.6133/ apjcn.2013.22.4.11.

8. Nea FM, Kearney J, Livingstone MB, Pourshahidi LK, Corish CA. Dietary and lifestyle habits and the associated health risks in shift workers. Nutr Res Rev. 2015;28(2):143-66.
9. Geliebter A, Gluck ME, Tanowitz M, Aronoff NJ, Zammit GK. Work-shift period and weight change. Nutrition. 2000;16(1):27-9.

10. Reeves SL, Newling Ward E, Gissane C. The effect of shiftwork on food intake and eating habits. Nutr Food Sci. 2004;34(5):216-21.

11. Bilski B. [Influence of shift work on the diet and gastrointestinal complains among nurses. A pilot study]. Med Pr. 2006;57(1):15-9. Polish.

12. Zhao I, Bogossian F, Song S, Turner C. The association between shift work and unhealthy weight: A cross-sectional analysis from the Nurses and Midwives' e-cohort Study. J Occup Environ Med. 2011;53(2):153-8, https://doi.org/ 10.1097/JOM.0b013e318205ele8.

13. Sahu S and Dey M. Changes in food intake pattern of nurses working raapidly rotating shift. Al Ameen J. Med. Sci. 2011;4(1):14-22.

14. Saksvik-Lehouillier I, Bjorvatn B, Hetland H, Sandal GM, Moen BE, Mageroy N, et al. Individual, situational and lifestyle factors related to shift work tolerance among nurses who are new to and experienced in night work. J Adv Nurs. 2013;69(5):1136-46, https://doi.org/10.1111/j.13652648.2012.06105.x.

15. Przeor M, Goluch-Koniuszy Z. Evaluation of nutrition and diet of nurses during peromenopause while working in shift system. Probl Hig Epidemiol. 2013;94(4):797-801.

16. Kim MJ, Son KH, Park HY, Choi DJ, Yoon CH, Lee HY, et al. Association between shift work and obesity among female nurses: Korean Nurses' Survey. BMC Public Health. 2013;13:1204, https://doi.org/10.1186/1471-2458-13-1204.

17. Naghashpour M, Amani R, Nematpour S, Haghighizadeh MH. Dietary, anthropometric, biochemical and psychiatric indices in shift work nurses. Food Nutr Sci. 2013; 4:1239-46, https://doi.org/10.4236/fns.2013.412158.

18. Tada Y, Kawano Y, Maeda I, Yoshizaki T, Sunami A, Yokoyama $\mathrm{Y}$, et al. Association of body mass index with lifestyle and rotating shift work in Japanese female nurses. Obesity (Silver Spring). 2014;22(12):2489-93, https://doi. org/10.1002/oby.20908.

19. Buchvold HV, Pallesen S, Oyane NM, Bjorvatn B. Associations between night work and BMI, alcohol, smoking, caffeine and exercise - A cross-sectional study. BMC Public Health. 2015;15:1112, https://doi.org/10.1186/s12889-0152470-2.

20. Ramin C, Devore EE, Wang W, Pierre-Paul J, Wegrzyn LR, Schernhammer ES. Night shift work at specific age ranges and chronic disease risk factors. Occup. Environ. Med. 2015; 72(2):100-7, https://doi.org/10.1136/oemed-2014-102292.

21. Yoshizaki T, Kawano Y, Noguchi O, Onishi J, Teramoto R, Sunami A, et al. Association of eating behaviours 
with diurnal preference and rotating shift work in Japanese female nurses: A cross-sectional study. BMJ Open. 2016;6(11):e011987, https://doi.org/10.1136/bmjopen-20 16-011987.

22. Hakim SA, Eldin WS, Mohsen A. Dietary behavior and its relation with lifestyle, rotating work shifts and job satisfaction among nurses of Ain Shams university hospitals. Egypt J Community Med. 2016;34(2):75-86.

23. Gladysz G, Kobos E, Czarnecka J, Imiela J. Nurses' eating habits in the workplace. Pieleg Pol. 2016;60(2):149-57.

24. Ajmajwal AM. Stress, shift duty, and eating behavior among nurses in Central Saudi Arabia. Saudi Med J. 2016; 34(2):191-8, https://doi.org/10.15537/smj.2016.2.13060.

25. Han K, Choi-Kwon S, Kim KS. Poor dietary behaviors among hospital nurses in Seoul, South Korea. Appl Nurs Res. 2016;30:38-44, https://doi.org/10.1016/j.apnr.2015. 10.009 .

26. Beebe D, Chang JJ, Kress K, Mattfeldt-Beman M. Diet quality and sleep quality among day and night shift nurses. J Nurs Manag. 2017;25(7):549-57, https://doi.org/10.11 11/jonm.12492.

27. Roskoden FC, Kruger J, Vogt LJ, Gartner S, Hannich HJ, Steveling A, et al. Physical activity, energy expenditure, nutritional habits, quality of sleep and stress levels in shift-working health care personnel. PLoS One. 2017;12(1): e0169983, https://doi.org/10.1371/journal.pone.0169983
28. Saksvik-Lehouillier I, Bjorvatn B, Hetland H, Sandal GM, Moen BE, Mageroy N, et al. Individual, situational and lifestyle factors related to shift work tolerance among nurses who are new to and experienced in night work. J Adv Nurs. 2013;69(5):113646, https://doi.org/10.1111/j.13652648.2012.06105.x.

29. Poole R, Kennedy OJ, Roderick P, Fallowfield JA, Hayes PC, Parkes J. Coffee consumption and health: Umbrella review of meta-analyses of multiple health outcomes. BMJ. 2017; 359:j5024, https://doi.org/10.1136/bmj.j5024.

30. Clark I, Landolt HP. Coffee, caffeine, and sleep: A systematic review of epidemiological studies and randomized controlled trials. Sleep Med Rev. 2017;31:70-8.

31. Subar AF, Freedman LS, Tooze JA, Kirkpatrick SI, Boushey C, Neuhouser ML, et al. Addressing current criticism regarding the value of self-report dietary data. J Nutr. 2015;145(12):2639-45, https://doi.org/10.3945/ jn.115.219634.

32. Park Y, Dodd KW, Kipnis V, Thompson FE, Potischman N, Schoeller DA, et al. Comparison of self-reported dietary intakes from the Automated Self-Administered 24-h recall, 4-d food records, and food-frequency questionnaires against recovery biomarkers. Am J Clin Nutr. 2018;107(1):80-93, https://doi.org/10.1093/ajcn/nqx002.

33. Gersovitz M, Madden JP, Smiciklas-Wright H. Validity of the 24-hr. dietary recall and seven-day record for group comparisons. J Am Diet Assoc. 1978;73(1):48-55.

This work is available in Open Access model and licensed under a Creative Commons Attribution-NonCommercial 3.0 Poland License - http://creativecommons.org/licenses/by-nc/3.0/pl/deed.en. 